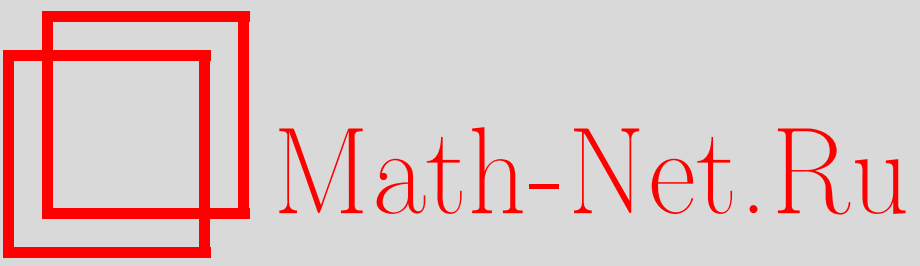

C. В. Матвеев, Табулирование трехмерных многообразий, УМH, 2005, том 60, выпуск 4, 97-122

DOI: https://doi.org/10.4213/rm1446

Использование Общероссийского математического портала Math-Net.Ru подразумевает, что вы прочитали и согласны с пользовательским соглашением

http://www.mathnet.ru/rus/agreement

Параметры загрузки:

IP : 3.85 .5 .30

26 апреля 2023 г., 14:49:00 


\title{
ТАБУЛИРОВАНИЕ ТРЕХМЕРНЫХ МНОГООБРАЗИЙ
}

\author{
C.B. Matbeeb
}

\begin{abstract}
Составление таблиц трехмерных многообразий требует разработки и компьютерной реализации двух алгоритмов: алгоритма перечисления и алгоритма распознавания. В статье излагаются теоретические основы этих алгоритмов и приводится обзор результатов по практическому табулированию трехмерных многообразий.

Библиография: 27 названий.
\end{abstract}

\section{СОДЕРЖАНИЕ}

1. Введение

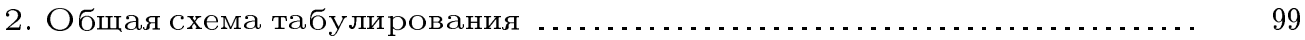

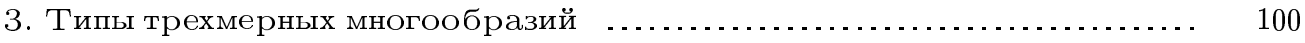

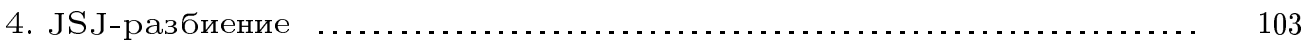

5. Табулирование с помощью спайнов ............................... 104

6. Распознавание многообразий …............................... 109

7. Результаты табулирования .................................... 112

8. Кристаллизации и расширенные диаграммы Хегора . ............... 118

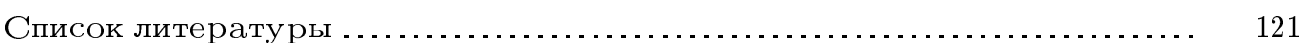

\section{1. Введение}

Трехмерное многообразие - это геометрический объект, локально устроенный так же, как и трехмерное евклидово пространство. Другими словами, каждая точка трехмерного многообразия должна иметь шаровую окрестность. Это понятие естественным образом обобщает понятие поверхности, когда каждая точка должна иметь круговую окрестность. Часто оказьвается удобньм рассматривать многообразия с краем, точкам которого разрешается иметь полушаровые окрестности. Край компактного трехмерного многообразия либо пуст (в этом случае многообразие назьвается замкнутым), либо является замкнутой поверхностью.

Работа выполнена при поддержке грантов РФФИ № 02-01-1013 и INTAS № 03-51-3663. Я благодарен также Математическому институту Макса Планка в Бонне, где был написан окончательный вариант статьи, за гостеприимство и финанасовую поддержку. 
Многообразия естественно появляются во многих областях математики, механики и физики, например, как множества решений систем уравнений. Другой источник появления многообразий - конфигурационные пространства различных механических систем. Поэтому не удивительно, что проблема классификации (и, в частности, табулирования) многообразий является одной из ключевых проблем современной математики.

Несколько наивное, хотя и правильное, объяснение важности задачи классификации трехмерных многообразий состоит в том, что мир, в котором мы живем, трехмерен. Более глубокая причина заключается в сушествовании тесных связей между трехмерной топологией и такими важными разделами науки, как математическая физика, статистическая механика, теория квантовых групп.

Хотя задача классификации трехмерных многообразий не решена до сих пор, усилия поколений математиков привели к доказательству ряда глубоких и содержательных результатов. В первую очередь, следует отметить построение явной классификации многообразий Зейферта и графф-многообразий Вальдхаузена. Другой важньй результат трехмерной топологии состоит в алгоритмической классификации неприводимых достаточно больших трехмерных многообразий, т.е. в построении алгоритма распознавания их гомеоморфности [4], [5]. Следует отметить, что, поскольку дополнительные пространства нетривиальных узлов и нераспадающихся зацеплений в трехмерном пространстве являются достаточно большими, небольшая модификашия упомянутого алгоритма приводит к алгоритмической классификации узлов и зацеплений.

В основе настоящего обзора лежит следуюшая естественная идея: нужно классифицировать многообразия в порядке возрастания их сложности - сначала самые простые, потом более сложные. Другими словами, задача заключается в составлении таблищ трехмерных многообразий, которые не содержат пропусков и дубликатов. Полезность таких таблиц подтверждается широким использованием аналогичных таблищ в теории узлов, но главный смысл состоит в том, что сама задача табулирования многообразий является хорошо поставленной интересной проблемой.

Следует отметить, что недавнее объявление Г. Перельмана о положительном решении геометризационной гипотезы Торстона (W. Thurston), частным случаем которой является знаменитая гипотеза Пуанкаре, способно только усилить интерес к составлению таблиц трехмерных многообразий. Согласно этой гипотезе, любое замкнутое трехмерное многообразие можно разрезать по двумерным торам на части, каждая из которых обладает однородной геометрической структурой одного из 8 типов. Насколько часто появляются геометрии каждого типа и как соответствующие геометрические многообразия склеиваются между собой? Как по заданному каким-нибудь способом трехмерному многообразию построить его разложение на геометрические части и как идентифицировать это многообразие с одним из известных? Табулирование (т.е. составление и анализ таблиц многообразий) как раз и помогает отвечать на эти и многие другие аналогичные вопросы.

Я начал серьезно заниматься табулированием трехмерных многообразий еше в 1972 г., когда эта тематика была доложена мною на семинаре Л.В.Келдьш и одобрена ею. Я благодарю В. В. Таркаева, которые не только написал все основные программы, но и принял непосредственное участие в разработке алгоритмов. Существенная 
помошь была оказана О.М. Давыдовым, Е.Л. Первовой, А.А. Перфильевьм, Е.А. Фоминых и другими участниками топологического семинара Челябинского государственного университета. Я благодарен А. Кавиккиоли (A. Cavicchioli), M.-P. Казали (M.-R. Casali), Б. Мартелли (В. Martelli) и K. Петронио (C. Petronio) за полезные обсуждения.

\section{2. Общая схема табулирования}

Для составления таблиц трехмерных многообразий какого-нибудь класса нужно разработать два алгоритма. Первый алгоритм должен перечислять многообразия, вернее, их комбинаторные (или иные) задания. Разумеется, вьполнение этого пункта нужно начать с описания используемых представлений. Второй алгоритм нужен для распознавания дубликатов, т.е. заданий, определяющих гомеоморфные многообразия. Если оба алгоритма найдены, то их совместное применение (перечисление и отсеивание дубликатов) позволяет предложить алгоритм построения классифицирующего списка, в которьй каждое многообразие входит только один раз и который рано или поздно включит в себя любое заранее заданное многообразие рассматриваемого класса. Именно в этом смысле говорят об алгоритмической классификации достаточно больших неприводимых трехмерных многообразий. Однако алгоритм распознавания таких многообразий, как и многие другие нетривиальные алгоритмы в трехмерной топологии, носит чисто теоретический характер и пока далек от практического применения. Поэтому составление таблиц требует разработки других, может быть, частичных, но намного более эффективных алгоритмов.

Обычно перечисление и табулирование многообразий ведется в порядке возрастания какого-нибудь параметра, характеризуюшего их сложность. Опыт показывает, что сложность геометрического объекта удобно оценивать числом его сингулярностей, а если объект однороден - то числом сингулярностей его минимального комбинаторного задания. Например, в качестве сложности графа обьчно берут число его вершин, а в качестве сложности узла - число двойных точек его минимальной диаграммы. При табулировании трехмерных многообразий чаще всего используются параметры, так или иначе связанные с числом тетраэдров в минимальных триангуляциях многообразий.

В каком виде выдавать ответ? Дело в том, что таблицы, состоящие из абстрактных комбинаторных кодов полученных многообразий (см., например, книгу [9]), имеют право на существование, но особого смысла в них нет. Хотелось бы иметь таблищы более приемлемого вида, по крайней мере, когда это возможно. Поэтому для многообразий, имеюших имена (т.е. входяших в уже классифицированные классы), распознавание и упоминание их имен и других характеристик обязательно.

Таким образом, при описании каждого конкретного способа табулирования нужно иметь в виду следуюшие моменты:

1) выбор способа задания многообразий;

2) выбор использованного понятия сложности;

3) описание алгоритма перечисления;

4) описание алгоритма распознавания; 
5) описание спектра допустимых имен и других характеристик.

Мы начнем с последнего пункта.

\section{3. Типы трехмерных многообразий}

3.1. Многообразия Зейферта. Самые простые примеры трехмерных многообразий - это прямые или косые произведения поверхностей на окружность. Разумеется, все они расслоены на окружности. Многобразия Зейферта получаются с помощью небольшой модификашии этой конструкции. При этом структура расслоения на окружности сохраняется, правда, появляются особые слои. Каждое ориентируемое многообразие Зейферта (для простоты мы ограничимся только такими) имеет вид $M=M\left(F ;\left(\alpha_{1}, \beta_{1}\right), \ldots,\left(\alpha_{n}, \beta_{n}\right)\right)$, где $F-$ компактная связная поверхность, назьваемая базой, $n$ - число особых слоев, а пары $\left(\alpha_{i}, \beta_{i}\right)$ взаимно простых целых чисел неприведенные параметры особых слоев. Неприведенность параметров означает, что мы не приводим $\beta_{i}$ по модулю $\alpha_{i}$. Удобно считать, что $\alpha_{i} \geqslant 1$. Многообразие $M$ получается из ориентируемого прямого или косого (но имеюшего сечение) произведения ориентируемой или, соответственно, неориентируемой поверхности $F$ на окружность следуюшим образом. Нужно удалить $n$ послойных полных торов и вклеить их назад по гомеоморфизмам, переводяшим меридианы в кривые типа $\left(\alpha_{i}, \beta_{i}\right)$, т.е. в кривые $\mu_{i}^{\alpha_{i}} \lambda_{i}^{\beta_{i}}$. Здесь через $\mu_{i}$ и $\lambda_{i}$ обозначены меридиан (край сечения) и параллель (слой), составляюшие каноническую систему координат на краевом торе.

Легко показать, что многообразие $M\left(F ;\left(\alpha_{1}, \beta_{1}\right), \ldots,\left(\alpha_{n}, \beta_{n}\right)\right)$ не меняется при следуюших операщиях над неприведенными параметрами:

1) перенумерация особых слоев;

2) смена знаков у всех параметров $\beta_{i}$ (эта операция отвечает смене ориентации многообразия);

$3)$ удаление или вставка слоя типа $(1,0)$; это соответствует переименованию особого слоя с указанньми параметрами в неособьй (каковым он и является), или наоборот;

4) замена двух пар параметров $\left(\alpha_{i}, \beta_{i}\right),\left(\alpha_{j}, \beta_{j}\right), i \neq j$, на пары $\left(\alpha_{i}, \beta_{i}+\alpha_{i}\right)$, $\left(\alpha_{j}, \beta_{j}-\alpha_{j}\right)$

$5)$ замена пары параметров $\left(\alpha_{i}, \beta_{i}\right)$ на пару $\left(\alpha_{i}, \beta_{i}+\alpha_{i}\right)$ при условии, что край многообразия не пуст; каноническая система координат $\mu, \lambda$ на одном из краевых торов заменяется при этом на систему $\mu \lambda, \lambda$.

Описанные операции (они называются торговлей параметрами) характеризуют послойную гомеоморфность многообразий Зейферта. Например, многообразия $M\left(S^{2} ;(2,1),(3,1),(5,-4)\right)$ и $M\left(S^{2} ;(2,-1),(3,1),(5,1)\right)$ послойно гомеоморфны. Многообразия $M\left(D^{2} ;(2,1),(3,-1)\right)$ и $M\left(D^{2} ;(2,1),(3,2)\right)$ также послойно гомеоморфны, но меридианы их канонических систем координат на крае отличаются на добавление параллели. Примеров гомеоморфных, но не послойно гомеоморфных многообразий Зейферта немного. Все они исчерпьваются следующим списком.

1. Каждое линзовое пространство $L(p, q)=M\left(S^{2} ;(q, p)\right)$ имеет много представлений в виде многообразий Зейферта, поскольку оно гомеоморфно любому такому многообразию $M\left(S^{2} ;\left(\alpha_{1}, \beta_{1}\right),\left(\alpha_{2}, \beta_{2}\right)\right)$, что $p=\alpha_{2} \beta_{1}-\alpha_{1} \beta_{2}, q=v \alpha_{1}-$ $u \beta_{1}$, а числа $u, v$ выбраны из условия $\alpha_{2} v-\beta_{2} u=1$. 
2. Многообразие $M\left(D^{2} ;(2,1),(2,-1)\right)$ гомеоморфно многообразию $M\left(M^{2}\right)=$ $M^{2} \tilde{\times} S^{1}$, где $M^{2}$ - лист Мёбиуса. Этот гомеоморфизм влечет еще два.

3. $M\left(S^{2} ;(2,1),(2,-1),(2,1),(2,-1)\right)=M\left(K^{2}\right)=K^{2} \tilde{\times} S^{1}$, где $K^{2} \tilde{\times} S^{1}$ - имеющее сечение косое произведение бутылки Клейна $K^{2}$ на окружность.

4. $M\left(\mathbb{R} P^{2} ;(\alpha, \beta)\right)=M\left(S^{2} ;(2,1),(2,-1),(\beta, \alpha)\right)$, где $\mathbb{R} P^{2}$ - проективная плоскость.

5. Разумеется, многообразие Зейферта над диском $D^{2}$ с одним особым слоем есть просто полньй тор: $M\left(D^{2} ;(\alpha, \beta)\right)=M\left(D^{2}\right)=D^{2} \times S^{1}$.

3.2. Граф-многообразия. Граф-многообразия были введены и классифицированы $\Phi$. Вальдхаузеном [27].

ОПРЕДЕЛЕНИЕ 1. Компактное ориентируемое трехмерное многообразие $M$ называется граф-многообразием, если его можно получить склеиванием нескольких экземпляров элементарных блоков $D^{2} \times S^{1}$ и $N^{2} \times S^{1}$, где $N^{2}$ - диск с двумя дырками, по некоторым гомеоморфизмам их краевых торов.

Легко показать, что любое ориентируемое многообразие Зейферта $M$ является графю-многообразием. Действительно, его базу $F$ можно разбить окружностями на блоки $D^{2}, N^{2}, M^{2}$. Переходя к прообразам этих блоков при проекции $p: M \rightarrow F$, мы получим разбиение многообразия $M$ на блоки $D^{2} \times S^{1}, N^{2} \times S^{1}, M^{2} \tilde{\times} S^{1}$. Остается вспомнить, что блок $M^{2} \tilde{\times} S^{1}$ гомеоморфен многообразию $M\left(D^{2} ;(2,1),(2,-1)\right)$, которое разбивается на два полных тора и один блок $N^{2} \times S^{1}$.

Итак, любое многообразие Зейферта можно собрать из элементарных блоков $D^{2} \times S^{1}, N^{2} \times S^{1}$ склеиванием по послойньм (т.е. переводящим слои в слои) гомеоморфизмам их граничных торов. При этом каждый блок $D^{2} \times S^{1}$ может быть разбит на слои по-разному, как многообразие Зейферта $M\left(D^{2} ;(\alpha, \beta)\right)$ для различных взаимно простых пар $\alpha, \beta$.

Графф-многообразия отличаются от многообразий Зейферта только тем, что склеиваюшие гомеоморфизмы не обязаны быть послойными. Поэтому не удивительно, что в их классификации явно участвуют многообразия Зейферта.

Теорема 1. Любое неприводимое граф-многообразие $W$ содержит такую систему $\mathscr{T}$ непересекающихся двумерных торов, что

1) $\mathscr{T}$ разбивает $W$ на многообразия Зейферта;

2) никакая меньшая подсистема системы $\mathscr{T ~ с в о и ̆ с т в о м ~ 1 ) ~ у ж е ~ н е ~ о б л а д а - ~}$ em.

Более того, система $\mathscr{T}$ определена однозначно с точностью до изотопии.

Из этой теоремы, которая представляет собой частньй случай теоремы о JSJ-разбиении (см. раздел 4) легко извлечь явную классификацию неприводимых графр-многообразий. Любое такое многообразие задается меченой молекулой, т.е. ориентированньм графом, каждой вершине (атому) которого сопоставлено многообразие Зейферта с указанньми базовой поверхностью и параметрами особых слоев. Торы на крае этого многообразия должны соответствовать ребрам, инцидентным рассматриваемой вершине, и на них должны быть введены канонические системы координат. Ребра графа должны быть помечены матрицами порядка 2, задаюшими гомеоморфизмы склейки. 
Суть теоремы классификации состоит в том, что две меченые молекулы задают одно и то же многообразие тогда и только тогда, когда с помошшю торговли параметрами особых слоев (с соответствуюшими изменениями канонических координат и матриц склеивания) и замен атома $M\left(D^{2} ;(2,1),(2,-1)\right)$ на атом $M^{2} \tilde{\times} S^{1}$ и обратно одну молекулу можно перевести в другую.

3.3. Геометрические многообразия. Трехмерное многообразие назьвается геометрическим, если его внутренность допускает полную локально-однородную риманову метрику. Однородность метрики означает, что любые две точки должны иметь изометричные окрестности. Согласно результатам У. Торстона, существует 8 типов геометрий: $E^{3}, S^{3}, S^{2} \times \mathbb{R}, H^{2} \times \mathbb{R}, \widehat{S L_{2} \mathbb{R}}, \mathrm{Nil}$, Sol и $H^{3}$, см. [21], [19]. Трехмерное многообразие может обладать не более чем одной из них. Наиболее интересны гиперболические многообразия, обладающие геометрией последнего типа. Они характеризуются тем, что их внутренности обладают полной римановой метрикой постоянной отрищательной кривизны -1 и поэтому локально изометричны пространству Лобачевского. Все компактные многообразия, обладающие геометриями оставшихся 7 типов, являются граф-многообразиями, причем многообразия с геометриями первых 6 типов являются многообразиями Зейферта.

Тип геометрии на замкнутом многообразии Зейферта $M\left(F ;\left(\alpha_{i}, \beta_{i}\right), 1 \leqslant i \leqslant n\right)$, можно определить по его числу Эйлера $e=-\sum_{i} \frac{\beta_{i}}{\alpha_{i}}$ и эйлеровой характеристике $\chi=\chi(F)-\sum_{i}\left(1-\frac{1}{\alpha_{i}}\right)$ его базы, рассматриваемой как орбифолд. Если $e=0$, то геометрия относится к типу $S^{2} \times \mathbb{R}$ при $\chi>0, E^{3}$ при $\chi=0$ и $H^{2} \times \mathbb{R}$ при $\chi<0$. Если $e \neq 0$, то геометрия имеет тип $S^{3}$ при $\chi>0$, Nil при $\chi=0$ и $\widetilde{S L_{2} \mathbb{R}}$ при $\chi<0$.

Замкнутое ориентируемое трехмерное многообразие $M$ обладает геометрией типа Sol, если выполнено одно из двух:

1. $M$ является многообразием Столлингса со слоем тор и гиперболической монодромией. Это означает, что $M$ получается из прямого произведения $T^{2} \times I$ двумерного тора на отрезок склеиванием оснований по гомеоморфизму, задаваемому такой матрицей $A=\left(\begin{array}{ll}a & b \\ c & d\end{array}\right)$, что $\operatorname{det}(A)=1$ и $|a+d|>2$.

2. $M$ получается склеиванием двух экземпляров утолщенной бутылки Клейна $K^{2} \times I$ и не является многообразием Зейферта. Здесь следует уточнить, что объединение двух утолшенных бутылок Клейна является многообразием Зейферта тогда и только тогда, когда в матрице гомеоморфизма склеивания, записанной в канонической системе координат на краемногообразия $K^{2} \times I=M\left(D^{2} ;(2,1),(2,-1)\right)$, имеется хотя бы один нуль. Наличие нуля означает, что слой одного из двух расслоений Зейферта на одной утолшенной бутылке Клейна переходит в слой одного из двух расслоений на второй утолщенной бутылке.

Перейдем к случаю гиперболических многообразий. Хотя примеры замкнутых трехмерных гиперболических многообразий появились довольно давно, первьй систематический способ их построения был предложен только в конце 70-х годов У. Торстоном.

Пусть $\stackrel{\circ}{Q}=Q \backslash \partial Q$ - гиперболическое многообразие с одним каспом (концом типа $\left.T^{2} \times[0, \infty)\right)$, являющееся внутренностью компактного многообразия $Q$ с краем тор. Выберем на торе $\partial Q$ систему координат и будем через $Q_{p, q}$ обозначать замкнутое многообразие, полученное заклейкой края полным тором по такому гомеоморфизму 
$h: \partial D^{2} \times S^{1} \rightarrow \partial Q_{p, q}$, что меридиан $\partial D^{2} \times\{*\}$ полного тора переходит в кривую типа $(p, q)$. Тогда оказывается, что для всех, кроме конечного числа, пар взаимно простых целых чисел $(p, q)$ замкнутое многообразие $Q_{p, q}$ является гиперболическим. При этом и многообразие, и структура на нем получаются взятием пополнения относительно специальным образом подобранной неполной гиперболической метрики на $Q$.

Таким образом, каждое гиперболическое многообразие с одним каспом порождает бесконечное семейство замкнутых гиперболических многообразий. Многообразия с одним каспом табулированы до сложности 7. В закодированном виде таблица входит в пакет программ SnapPea Дж. Викса (J. Weeks). Bсе спешиальные спайны с 2 и 3 вершинами гиперболических многообразий с одним каспом изображены в [16] (определение специального спайна и сложности многообразия см. в разделе 5). Они задают 11 различных гиперболических многообразий, которые мы будем обозначать $Q_{i}$. Многообразие $Q_{2}$ - это дополнительное пространство узла восьмерки, знаменитое тем, что именно с него У. Торстон начал свои исследования по построению замкнутых гиперболических многообразий пополнением неполных пространств. Сложность этого многообразия равна двум. Его "близнец" $Q_{1}$ тоже имеет сложность 2 и является дополнительным пространством некоторого узла в линзе $L(5,1)$. См. также [3], где приведены так называемые обезвоженные описания идеальных триангуляий гиперболических многообразий с 4 и менее тетраэдрами.

Наличие метрики позволяет вычислять объемы гиперболических многообразий. Объемы замкнутых многообразий и многообразий с каспами конечны. Известно (см. [23]), что множество всех точек действительной оси $\mathbb{R}$, отвечающих объемам ориентируемых гиперболических многообразий, имеет вид $\omega^{\omega}$. Отсюда следует, что существует первый (минимальный) объем, затем второй объем, затем третий, и т. д., пока не появится первая предельная точка. Она отвечает минимальному объему для многообразий с одним каспом. После чего ситуашия повторяется: появляются предельные точки второго порядка, третьего и т. д.

Упомянутое вьше многообразие $Q_{1}$ замечательно тем, что одно из его заполнений Дена имеет наименьший объем среди всех известных замкнутых ориентируемых гиперболических многообразий, см. [28], [18].

\section{JSJ-разбиение}

Разбиение, упомянутое в заголовке этого раздела, названо по первым буквам фамилий открывших его математиков (Jaco, Shalen, Johannson, cм. [6]-[8]).

ОПРЕДЕЛЕнИЕ 2 . Замкнутая поверхность $F$ внутри трехмерного многообразия $M$ называется несжимаемой, если край любого 2 -диска $D \subset M$, пересекаюшего $F$ ровно по своему краю, тривиален, т.е. ограничивает диск в $F$.

Для двусторонних поверхностей несжимаемость эквивалентна $\pi_{1}$-инъективности поверхности: индуцируемый вложением гомоморфизм $i_{*}: \pi_{1}(F) \rightarrow \pi_{1}(M)$ фундаментальных групш должен иметь тривиальное ядро.

Будем говорить, что несжимаемый тор $T$ в замкнутом неприводимом ориентируемом трехмерном многообразии $M$ является груббцм, если любой другой несжимаемый тор в $M$ изотопен такому тору $T^{\prime}$, что $T \cap T^{\prime}=\varnothing$. 
ОПРЕДЕЛЕНИЕ 3 . Конечная система $\mathscr{S}$ непересекаюшихся торов в замкнутом неприводимом ориентируемом трехмерном многообразии $M$ назьвается JSJ-cuстемой, если выполнены следуюшие условия:

1) все торы являются грубыми;

2) среди них нет изотопных торов;

3) $\mathscr{S}$ максимальна среди систем, обладаюших свойствами 1), 2); это означает, что любая другая система $\mathscr{S}^{\prime} \supset \mathscr{S}$ со свойствами 1$), 2$ ) должна быть изотопной системе $S$.

ТЕОРема 2. Для любого замкнутого неприводимого ориентируемого трехмерного многообразия $M$ JSJ-система существует и единственна с точностью до изотопии.

Идея доказательства этой теоремы проста. Существование максимальной системы следует из теоремы конечности Кнезера-Хакена о том, что число попарно не изотопных несжимаемых поверхностей в неприводимом трехмерном многообразии ограничено некоторой константой, зависящей только от числа тетраэдров в триангуляции многообразия. Единственность получается применением свойства грубости: торы второй JSJ-системы можно сдвинуть со всех торов первой, а тогда изотопность систем будет следовать из их максимальности.

Торы JSJ-системы разбивают многообразие на части, которые мы будем назьвать камерами. Важность теоремы 2 состоит в том, что геометрическая структура камер допускает точное описание.

ТЕОРема 3. Если JSJ-система замкнутого связного неприводимого ориентируемого трехмерного многообразия $M$ не пуста, то каждая камера является либо многообразием Зейферта, либо гиперболическим многообразием.

Тип камеры зависит от того, содержит ли она не параллельное краю несжимаемое кольцо, край которого лежит на крае камеры. Если такое кольцо имеется, то максимальность JSJ-системы позволяет распространить его стандартное расслоение на окружности до расслоения Зейферта на всей камере. Если такого кольца нет, то гиперболичность камеры следует из справедливости геометризационной гипотезы Торстона для достаточно больших многообразий.

Теоремы 2, 3 позволяют использовать язык меченых молекул для задания произвольных трехмерных многообразий, а не только граф̆-многообразий. Единственное отличие состоит в том, что атомы могут быть гиперболическими многообразиями и системы координат на краях гиперболических частей нужно указывать отдельно.

\section{5. Табулирование с помощью спайнов}

5.1. Триангуляции и спайны. Пусть топологическое пространство $M$ получено склеиванием треугольных граней нескольких ориентированных тетраэдров по обращаюшим ориентацию аффинным гомеоморфизмам так, что каждая грань отождествляется ровно с одной другой гранњю. Хорошо известно, что $M$ является замкнутым ориентируемьм трехмерным многообразием тогда и только тогда, когда его эйлерова 
характеристика $\chi(M)$ равна нулю. В этом случае говорят о сингулярной триангуляиии многообразия $M$. От обычной триангулящии она отличается тем, что различные тетраэдры могут пересекаться не по одной, а по нескольким граням.

Если $\chi(M) \neq 0$, то $M$ является многообразием с коническими особенностями, т.е. получается из некоторого компактного трехмерного многообразия $M^{\prime}$ с краем взятием конусов над всеми компонентами края. При этом вершины конусов получаются склеиванием вершин тетраэдров. Если их удалить, то получится идеальная триангуляиия некомпактного многообразия $M^{\prime} \backslash \partial M^{\prime}$. С другой стороны, $M^{\prime}$ можно рассматривать как результат склеивания тетраэдров с обрезанными уголками.

Описанные типы триангулящй удобны для задания многообразий, как компактных, так и некомпактных: достаточно явно указать, как склеиваются грани и какой тип триангуляции имеется в виду.

Двойственьй способ задания многообразий состоит в использовании специальных спайнов. Пусть $M$ - трехмерное многообразие с краем. Наличие края не является ограничением, так как если многообразие замкнуто, то из него всегда можно вырезать трехмерный шар и получить новое многообразие с краем сфера. Будем, начиная с края, сжимать (или сдавливать) $M$ внутрь до тех пор, пока это возможно. В итоге мы получим полиэдр размерности 2 или меньше, которьй и называется спайном. Строгое определение таково: подполиэдр $P \subset M$ назьвается спайном, если разность $M \backslash P$ гомеоморфна прямому произведению $\partial M \times(0,1]$. Спайн замкнутого многообразия $M$ определяется как спайн многообразия $M$, из которого удален открытый трехмерньй шар.

ОПРЕДЕЛЕНИЕ 4. Полиэдр $P$ назьвается специальньлм, если на нем можно ввести структуру клеточного комплекса так, что линк каждой вершины является полным графом с 4 вершинами.

Типичная особенность специального полиэдра (будем называть ее бабочкой) изображена на рис. 1. Тело бабочки состоит из четырех отрезков с обшим концом, причем на каждые два отрезка натянуто одно крыло.

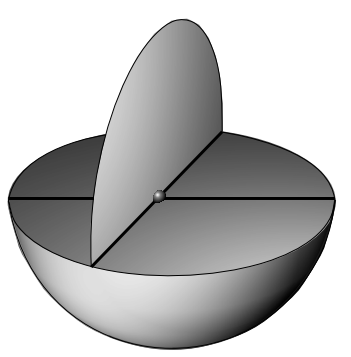

Рис. 1. Бабочка

Объединение особых точек (вершин и тройных линий) специального полиэдра $P$ называется его особылм графом и обозначается $S(P)$. Отметим, что особьй графи любого специального полиэдра является регулярным графом валентности 4. Это означает, что из каждой его вершины исходит ровно 4 ребра.

Далеко не все специальные полиэдры утолщаемы, т.е. являются спайнами ориентируемых трехмерных многообразий. Простой критерий утолшаемости таков: 
специальный полиэдр $P$ утолшаем тогда и только тогда, когда регулярная окрестность $N_{P}(S(P))$ в полиэдре $P$ его особого графа $S(P)$ вкладьвается в $\mathbb{R}^{3}$. Это дает полезньй способ изображения специальных спайнов: мы просто рисуем окрестность $N_{P}(S(P))$. По этой окрестности и спайн $P$, и исходное трехмерное многообразие восстанавливаются однозначно. На рис. 2 изображены специальный спайн $P$ стандартного трехмерного шара (дом Бинга с двумя комнатами) и вложение окрестности $N_{P}(S(P))$ в $\mathbb{R}^{3}$. Ребра спайна ориентированы и пронумерованы для того, чтобы ввести удобньй способ численного задания спайна: нужно указать, как граничные кривые 2-клеток проходят по ребрам.

1

2

$\begin{array}{llllllllll}1 & 3 & 2 & -3 & 4 & 2 & -4 & -1 & 4 & -3\end{array}$

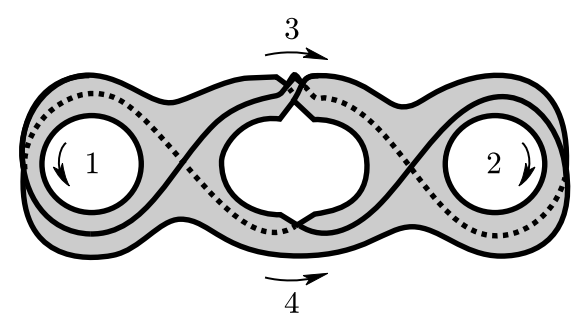

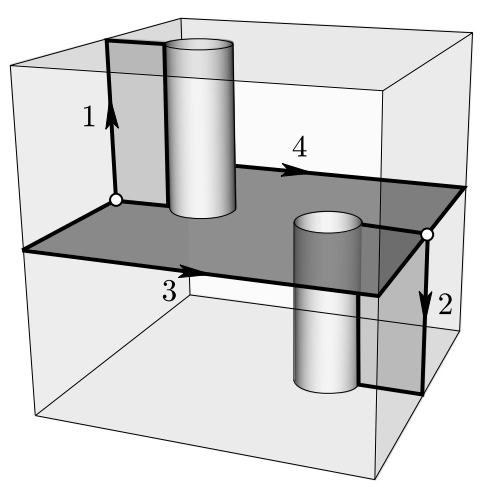

Рис. 2. Дом Бинга с двумя комнатами

Упомянутая вьше двойственность между сингулярными и идеальными триангуляциями и специальными спайнами состоит в следуюшем. Поместим в стандартньй тетраэдр с обрезанными уголками бабочку так, как это изображено на рис. 3. Тогда при склеивании тетраэдров в многообразие бабочки склеиваются в специальньй спайн этого многообразия. Обратно, если каждую вершину специального спайна утолшить до тетраэдра с обрезанными уголками и склеить тетраэдры так, как показывает спайн, то получится определяемое им многообразие.

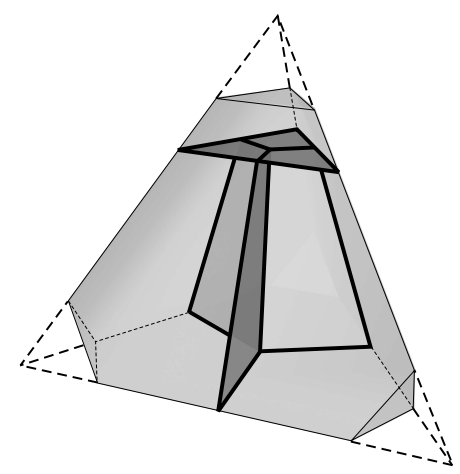

Рис. 3. Бабочка в тетраэдре 
5.2. Сложность многообразий. При работе со специальньми спайнами приходится выполнять довольно грубые операции, например, прокальвать и коллапсировать 2-клетки и разрезать спайн на части. Такие операции не сохраняют свойство полиэдра быть специальным. Поэтому удобно ввести более широкий класс полиэдров.

ОПРеДЕЛЕниЕ 5 . Компактный полиэдр $P$ назьвается nростылм, если его можно вложить в некоторый специальньй полиэдр.

Эквивалентное определение: полиэдр прост, если некоторая окрестность каждой его точки вкладывается в бабочку. Отсюда следует, что простой полиэдр может иметь одномерную часть, а также ребра, к которым примыкает меньше трех клеток (одна или две). Под истинной вершиной простого полиэдра понимается точка, регулярная окрестность которой является бабочкой.

ОПРЕДЕЛЕНИЕ 6. Сложность $c(M)$ компактного трехмерного многообразия $M$ равна $k$, если оно имеет простой спайн с $k$ истинными вершинами и не имеет простых спайнов с меньшим числом истинных вершин.

Сложность $c(M)$ обладает рядом полезных свойств.

1. Сложность аддитивна по отношению к связной сумме многообразий: $c\left(M_{1} \# M_{2}\right)=c\left(M_{1}\right)+c\left(M_{2}\right)$, см. [13], [14].

2. Для любого числа $k$ имеется только конечное число связных замкнутых неприводимых ориентируемых многообразий сложности $k$. Аналогичное свойство конечности сохраняется и для некоторых многообразий с краем, например, для неприводимых многообразий без собственных несжимаемых колец, не параллельных краю.

3. При разрезании многообразия по несжимаемой поверхности его сложность не увеличивается. Это вполне соответствует наблюдениям топологов, которые часто использовали разрезания для упрощения многообразия.

4. Все замкнутые ориентируемые трехмерные многообразия сложности $\leqslant 8$ являются граф-многообразиями [13].

5.3. Перечисление спайнов. Идея перечисления спайнов с данньм числом вершин $n$ проста. Сначала нужно перечислить все связные регулярные графы валентности 4 , которые имеют $n$ вершин. Затем каждьй граф поместить в $\mathbb{R}^{3}$ и заменить все его вершины на бабочки, см. рис. 4. При этом нужно учитьвать, что каждую вершину можно заменить на бабочку двумя способами, чтобы использовать обе возможные ориентации отвечающего ей тетраэдра. Наконец, для каждого ребра нужно перебрать все три возможных склеивания бабочек на его концах и заклеить дисками окружности на крае каждого получившегося полиэдра. Разумеется, мы получим все специальные спайны с $n$ вершинами. Однако здесь мы сталкиваемся с рядом проблем.

1. Число регулярных графов с $n$ вершинами растет очень быстро: как показали компютерные эксперименты, с каждым шагом оно увеличивается более чем втрое.

2. Число спайнов, смоделированных на регулярном графе с $n$ вершинами, растет также очень быстро, как $2^{n-1} 3^{2 n}$. 
3. Среди многообразий, отвечающих построенньм спайнам, много лишних. K их числу мы относим приводимые многообразия (например, нетривиальные связные суммы) и дубликаты (гомеоморфные копии одного и того же многообразия). Причина появления дубликатов двояка: во-первых, многообразие, которое впервые появляется для какого-нибудь числа вершин $n$, повторяется и для бо́льших значений $n$. Это происходит потому, что конструируемые спайны не обязаны быть минимальньми. Во-вторых, одно и то же многообразие может иметь несколько различных минимальных специальных спайнов.

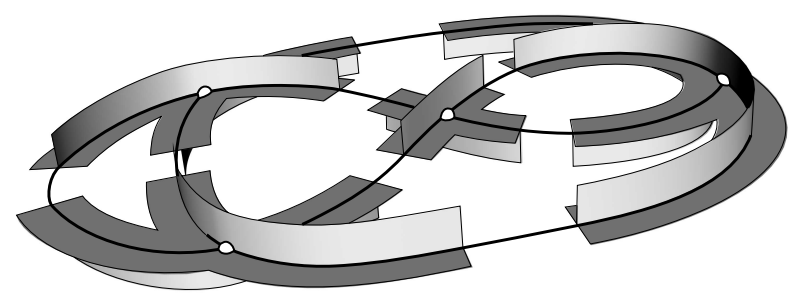

Рис. 4. Как строить специалные спайны с данньм особым графом

Естественный путь решения этих проблем состоит в раннем обнаружении и отбрасьвании графов и спайнов, которые дают лишние многообразия. Мы ограничимся несколькими примерами для случая, когда рассматриваемые трехмерные многообразия замкнуты, неприводимы и ориентируемы.

ПримеР 1. Допустим, что регулярный граф $G$ содержит тройное ребро или отличное от петли ребро, каждый конец которого являются разбивающей вершиной (т.е. после удаления этого конца граф становится несвязным). Тогда граф $G$ можно отбросить, поскольку ему не соответствует ни одного минимального спайна замкнутого многообразия.

ПримеР 2. Допустим, что граничная кривая одной из 2-компонент специального спайна $P$ имеет противоход, т.е. проходит по одному ребру дважды в противоположных направлениях. Тогда отвечаюшее этому спайну многообразие можно отбросить, поскольку оно либо не замкнуто, либо приводимо, либо имеет специальный спайн с меньшим числом вершин.

ПримеР 3. Допустим, что специальньй спайн $P$ имеет короткую граничная кривую, т.е. что граничная кривая одной из его 2 -клеток проходит через $k \leqslant 3$ вершины, причем через каждую из них ровно один раз. Тогда соответствующее многообразие можно отбросить по той же причине.

Применение уже этих и других критериев позволило резко уменьшить число получаюшихся спайнов. Например, на уровне сложности 5 вместо порядка $10^{7}$ потенциально существуюших специальных спайнов с 5 вершинами остается около 100 , а на уровне сложности 10 вместо порядка $10^{16}$ спайнов остается около 30000 . Для сравнения стоит упомянуть окончательное число многообразий: 31 на сложности 5 и 3078 на сложности 10. 
Нужно отметить, что эти критерии можно применять уже в процессе построения графа или спайна. Если, скажем, при склеивании всего нескольких бабочек получается противоход, то спайн бракуется и склейки на других ребрах не рассматриваются.

Хороший способ перечисления спайнов и многообразий был предложен Б. Мартелли и К. Петронио. Уже анализ таблицы специальных спайнов многообразий сложности 6 (см. [15], [16]) показьвает, что все они состоят из сравнительно небольшого числа стандартных блоков. Это наблюдение было использовано М. Овчинниковым при составлении таблищы многообразий сложности 7. Б. Мартелли и К. Петронио пошли гораздо дальше: они построили красивую теорию разбиений многообразий на элементарные блоки, которая работает даже для многообразий с тривиальным JSJ-разбиением, см. [11]. Число элементарных блоков, достаточных для сборки любого замкнутого ориентируемого неприводимого многообразия сложности $\leqslant 9$ удивительно мало: нужно всего около десятка блоков для сборки 1135 из 1154 таких многообразий. Оставшиеся 19 многообразий имеют тривиальные разбиения в смысле Мартелли-Петронио и поэтому сами являются элементарными блоками. Более того, знание элементарных блоков позволяет не только собирать многообразия, но и одновременно определять типы многообразий (т.е. присваивать им имена).

\section{6. Распознавание многообразий}

Идея распознавания многообразия, заданного своим специальньм спайном, состоит в следуюшем. Будем упрощать спайн с помощью различных преобразований до тех пор, пока это возможно. При этом многообразие может меняться, но контролируемым образом. Информация, необходимая для восстановления многообразия, должна сохраняться. Дадим краткое описание распознавания, предполагая, что край рассматриваемого многообразия $M$ состоит из сфер и торов.

Первая трудность состоит в том, что приходится рассматривать не только специальные, но и простые спайны, которые в отличие от специальных могут утолшаться до трехмерного многообразия неоднозначно. Поэтому простые спайны приходится рассматривать вместе с их утолщениями. Удобньй способ состоит в том, что трехмерное многообразие $M$ задается как

- ориентированный клеточный комплекс $P$, задаюший простой спайн многообразия $M$;

- ориентированная поверхность $\partial M$, также представленная в виде клеточного комплекса;

- заданное соответствием между клетками клеточное отображение $p: \partial M \rightarrow P$.

Всего имеется около двух десятков различных приемов упрощения спайна. Мы приведем три наиболее эффективных приема.

1. Разрезание по дуге. Допустим, что спайн $P$ связного многообразия $M$ содержит двумерную клетку с двумя различными свободными ребрами. Тогда мы разрезаем спайн по дуге, соединяющей середины этих ребер. При этой операции многообразие $M$ меняется: новое многообразие $M_{1}$ получается из него разрезанием ручки индекса 1 . Несложный анализ показывает, что тогда либо $M_{1}$ состоит из двух компонент, граничная связная сумма которых есть $M$, либо $M$ получается из одного из многообразий $M_{1} \# S^{2} \times S^{1}, M_{1} \# D^{2} \times S^{1}$ заклеиванием шаром сферической компоненты края. 
2. Разрезание по окружности. Допустим, что спайн $P$ многообразия $M$ содержит окружность $C$, которая не стягиваема в $M$, лежит в несингулярной части спайна $P$ (представляющей собой объединение поверхностей) и сохраняет ориентацию. Тогда мы разрезаем спайн по $C$. При этом новое многообразие $M_{1} \subset M$ получается из многообразия $M$ разрезанием по несжимаемому кольцу $A=C \times I$ и разность $M \backslash M_{1}$ есть либо $N^{2} \times S^{1}$, либо $M_{0}^{2} \tilde{\times} S^{1}$, где $N^{2}$ - диск с двумя дырками и $M_{0}^{2}-$ лист Мёбиуса с одной дыркой. На рис. 5 показана двумерная версия этого эффекта. Трехмерную иллюстрацию можно получить взятием прямого или косого произведения изображенных картинок на окружность.
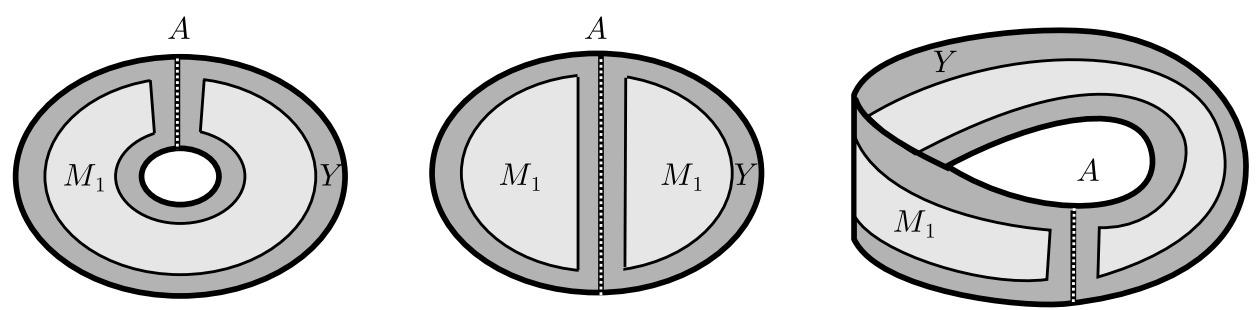

Рис. 5. $Y=M \backslash M_{1}$ есть либо $N^{2} \times S^{1}$, либо $M_{0}^{2} \tilde{\times} S^{1}$

3. Прокалывание двумерной клетки. Допустим, что многообразие $M$ замкнуто, а край утолшения спайна $P$ состоит из одной сферы. Тогда мы прокальваем одну из 2-клеток спайна $P$ (т.е. вырезаем из нее маленький диск). Многообразие $M$ и новое многообразие $M_{1}$ связаны так: $M$ получается из $M_{1}$ приклеиванием полного тора.

При каждом из описанных разрезаний спайн становится проше, так как в процессе последуюшего коллапсирования исчезает его существенная часть. Как эти и другие упрошаюшие преобразования помогают распознать многообразие $M ?$ Дело в том, что при упрощениях происходит рост оснашенной молекулы, задающей $M$. В начальньй момент молекула состоит из одного нераспознанного атома с выходящими из него виртуальными ребрами, символизирующими торические компоненты его края. При разрезании по дуге этот атом распадается на два отдельных атома. При разрезании по окружности появляется либо тройная вершина (атом типа $N^{2} \times S^{1}$ ) либо мёбиусова вершина (атом типа $M_{0}^{2} \tilde{\times} S^{1}$ ). Наконец, при прокальвании клетки от атома отщепляется вершина типа полного тора. При этом ребра растущей молекулы снабжаются матрицами порядка 2, задающими гомеоморфизмы склеивания, а появляющиеся новые нераспознанные атомы задаются указанием их спайнов, см. рис. 6 .

Важное замечание: так как все используемые операции упрощают спайн, то процесс построения оснашенной молекулы заведомо закончится.

Предположим, что все атомы получившейся молекулы оказались элементарнылми (т.е. уже распознанными известными многообразиями, см. ниже). Тогда начинается завершающий этап распознавания. Он заключается в обратной сборке молекулы в исходное многообразие, при которой все получающиеся многообразия (в том числе, $M$ ) снабжаются именами.

Разумеется, число элементарных атомов (әлементарных кирпичей) зависит от сложности $n$ многообразий, подлежаших распознаванию. При $n \leqslant 8$ достаточно все- 

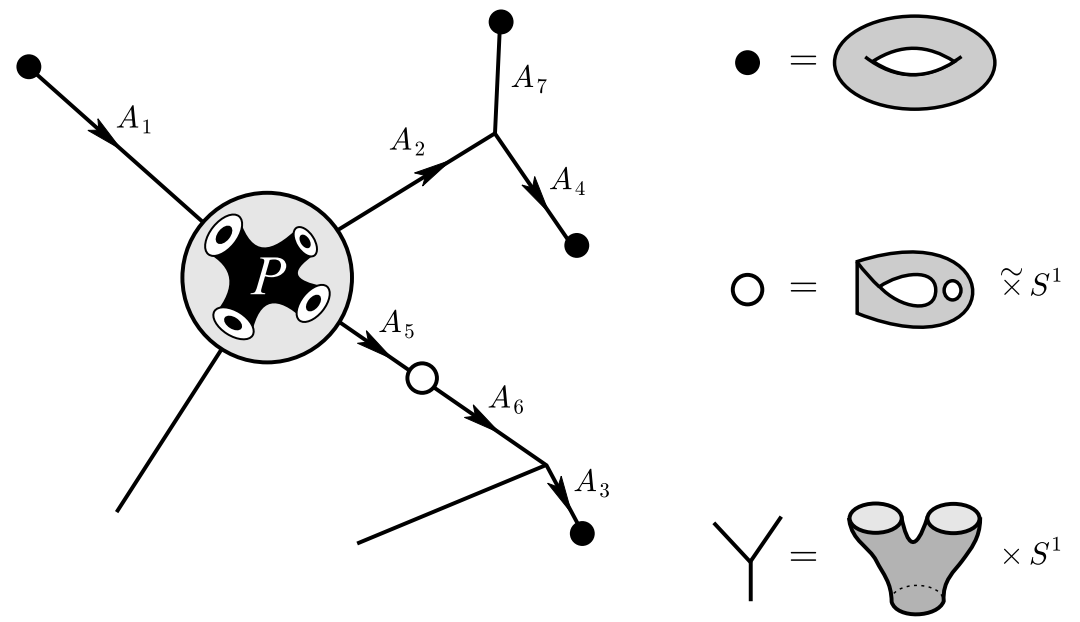

Рис. 6. Оснащенная молекула

го двух атомов $D^{2} \times S^{1}$ и $N^{2} \times S^{1}$. Из них можно собрать только графф-многообразия. При больших $n$ нужны дополнительные кирпичи, которые выявлялись, распознавались и включались в число элементарных в процессе табулирования. Оказалось, что при $n=9$ достаточно двух дополнительных кирпичей $Q_{1}, Q_{2}$ сложности 2, при $n=10$ нужны все девять кирпичей $Q_{3}, \ldots, Q_{11}$ сложности 3 , а при $n=11$ - еше 12 кирпичей сложности $\leqslant 5$. Каждый такой кирпич является гиперболическим многообразием с одним каспом.

Процесс обратной сборки молекулы состоит в последовательном применении всего двух операций. Будем называть атом зейфертовым, если таковьм является отвечающее ему многообразие.

Присоединение полного тора. Допустим, что молекула предписьвает приклеить полный тор к другому зейфертову атому по матрище $A$, в верхнем левом углу которой стоит не нуль. Условие на матрицу означает, что меридиан полного тора переходит в кривую, не изотопную слою расслоения Зейферта. Тогда мы выполняем предписание: объединяем зейфертов атом и полньй тор в новьй зейфертов атом. База нового атома получается из базы старого приклеиванием диска, причем к старым особым слоям добавляется новый слой, параметры которого составляют первый столбец матрицы.

Обгединение двух зейфертовых атомов. Допустим, что молекула предписьвает склеить два отличных от полного тора зейфертовых атома по матрице, в верхнем правом углу которой стоит нуль. Это означает, что при склейке слои переходит в слои. Тогда мы объединяем атомы в один зейфертов атом. Базы атомов склеиваются по одной краевой окружности, а особые слои объединяются.

Разумеется, при вьполнении этих операций нужно помнить про возможность замены многообразия $M\left(D^{2} ;(2,1),(2,-1)\right)$ многообразием $M^{2} \tilde{\times} S^{1}$ и обратно. Применяя указанные операции до тех пор, пока это возможно, мы получим так назьваемую приведенную молекулу, атомами которой являются многообразия Зейферта и элементарные атомы, не являющиеся зейфертовьми. Фактически, эта молекула и дает ответ на поставленную задачу распознавания. Потенциально возможны следуюшие случаи. 
1. Если все атомы приведенной молекулы окажутся зейфертовьми, то получается граф-многообразие с известными параметрами. В частности, если приведенная молекула содержит только один атом и не имеет петель, то получится многообразие Зейферта, а если останется один атом $T^{2} \times I$ с одним ребром-петлей, то тип многообразия (зейфертово или “operatorname Sol) определяется по матрище монодромии.

2. Если приведенная молекула состоит из гиперболического атома типа $Q_{i}$ и атома $D^{2} \times S^{1}$, соединенных одним ребром, то исходное многообразие имеет вид $\left(Q_{i}\right)_{p, q}$, где параметры $p, q$ вычисляются исходя из матрищы склеивания.

3. Приведенная молекула может содержать как другие зейфертовы и гиперболические атомы, так и неизвестные (т.е. еще не распознанные) атомы. В этом случае ответ на задачу распознавания носит условньй характер, по модулю распознавания входящих в нее неизвестных атомов.

В результате обширного компьютерного эксперимента выяснилось, что программа, основанная на указанных принципах, распознает многообразия довольно хорошо. В частности, она распознала все замкнутые ориентируемые неприводимые многообразия до сложности 11 включительно.

\section{7. Результаты табулирования}

\section{1. Таблица и графики роста.}

ТЕОРема 4. Число замкнутых ориентируемых неприводимых трехмерных многообразий сложности $\leqslant 11$ задается табличей 1.

ТАБЛИЦА 1

\begin{tabular}{|c|c|c|c|c|c|c|c|c|}
\hline Тип $\backslash c$ & $\leqslant 5$ & 6 & 7 & 8 & 9 & 10 & 11 & Итого \\
\hline$S^{3}$ & 61 & 61 & 117 & 214 & 414 & 798 & 1582 & 3247 \\
\hline$E^{3}$ & 0 & 6 & 0 & 0 & 0 & 0 & 0 & 6 \\
\hline $\mathrm{Nil}$ & 0 & 7 & 10 & 14 & 15 & 15 & 15 & 76 \\
\hline$H^{2} \times \mathbb{R}$ & 0 & 0 & 0 & 2 & 0 & 8 & 4 & 14 \\
\hline$\widetilde{S L_{2} \mathbb{R}}$ & 0 & 0 & 39 & 162 & 513 & 1416 & 3696 & 5826 \\
\hline Sol & 0 & 0 & 5 & 9 & 23 & 39 & 83 & 159 \\
\hline$H^{3}$ & 0 & 0 & 0 & 0 & 4 & 25 & 120 & 149 \\
\hline Составные & 0 & 0 & 4 & 35 & 185 & 777 & 2921 & 3922 \\
\hline Итого & 61 & 74 & 175 & 436 & 1154 & 3078 & 8421 & 13399 \\
\hline
\end{tabular}

Многообразия сложности $c \leqslant 5$ были классифицированы С.В. Матвеевым и В. В. Савватеевым [12] еше в 1974 г. Перечислялись они с помошюю компьютера, а распознавались вручную. Как видно из таблицы, все они являются эллиптическими 
многообразиями. При этом 44 многообразия из 61 являются линзами. Распределение многообразий по сложности таково:

\begin{tabular}{|c|c|c|c|c|c|c|c|}
\hline$c$ & 0 & 1 & 2 & 3 & 4 & 5 & Итого \\
\hline$N(c)$ & 3 & 2 & 4 & 7 & 14 & 31 & 61 \\
\hline
\end{tabular}

Позднее С. В. Матвеевым были табулированы многообразия сложности 6 [15]. Тот же самый метод был использован М. А. Овчинниковым [20] для составления таблицы для сложности 7. Распознавались многообразия по-прежнему вручную, хотя и усовершенствованньм методом (с выделением и использованием элементарных блоков). Следующий шаг был сделан Б. Мартелли, которьй не только независимо от М. А. Овчинникова заметил полезность элементарных блоков, но и совместно с К. Петронио [11] разработал стройную законченную теорию, сушественно упростившую табулирование и распознавание. За счет этого им были составлены таблицы ориентируемых многообразий до сложности 9 и исправлена ошибка М. А. Овчинникова, которьй не заметил гомеоморфности двух многообразий сложности 7. Элементы ручного распознавания остались. Многообразия сложности 10 были табулированы Б. Мартелли [10], которьй использовал прежний метод, и С. В. Матвеевым [17], которьй распознавал многообразия с помощью компьютерной программы, написанной по его алгоритму В.В. Таркаевьм. Сравнение промежуточных результатов помогло устранить некоторые недочеты как метода Б. Мартелли, так и алгоритма С. В. Матвеева. В конце концов результаты совпали для всех сложностей $\leqslant 10$. Наконец, последний шаг был сделан С. В. Матвеевым и В. В. Таркаевым, которые с помощью усовершенствованной программы табулировали все замкнутые неприводимые ориентируемые трехмерные многообразия сложности 11 (см. [17]). Многообразия сложности 12 тоже перечислены, но отсутствие дубликатов пока не проверено. Поэтому соответствуюшие числа в таблицу не включены.

На рис. 7 рост числа многообразий разных типов показан граффически. Поскольку все многообразия с геометриями $S^{3}, E^{3}, \mathrm{Nil}, H^{2} \times \mathbb{R}, \widetilde{S L_{2} \mathbb{R}}$ являются многообразиями Зейферта, данные о них представлены одним графиком (оба замкнутых ориентируемых многообразия $\left(S^{2} \times S^{2}\right.$ и $\left.\mathbb{R} P^{3} \# \mathbb{R} P^{3}\right)$ с геометрией $S^{2} \times \mathbb{R}$ приводимы, поэтому они не влияют ни на таблищы, ни на графики) Неясности с дубликатами сложности 12 на вид графиков никак не влияют. По оси ординат выбрана логарифмическая шкала с основанием 2.

7.2. Комментарии к таблице. Первое заключение, которое можно сделать после просмотра таблицы и информации про конкретные многообразия, состоит в том, что никаких неожиданностей в ней нет. Все так, как это предсказьвается теорией: каждое многообразие либо является геометрическим, либо разбивается на геометрические. При этом сложность многообразий растет, когда они усложняются в неформальном смысле этого слова.

Многообразия сложности 0 таковы: $S^{3}, \mathbb{R} P^{3}$ и линза $L(3,1)$. Оба многообразия сложности 1 являются линзовыми пространствами. Первое многообразие, не входящее в класс линзовых пространств, появляется на уровне сложности 2 . Это - фактор-пространство трехмерной сферы по группе единищ кватернионов $Q_{8}=\{ \pm 1, \pm i$, $\pm j, \pm k\}$. 


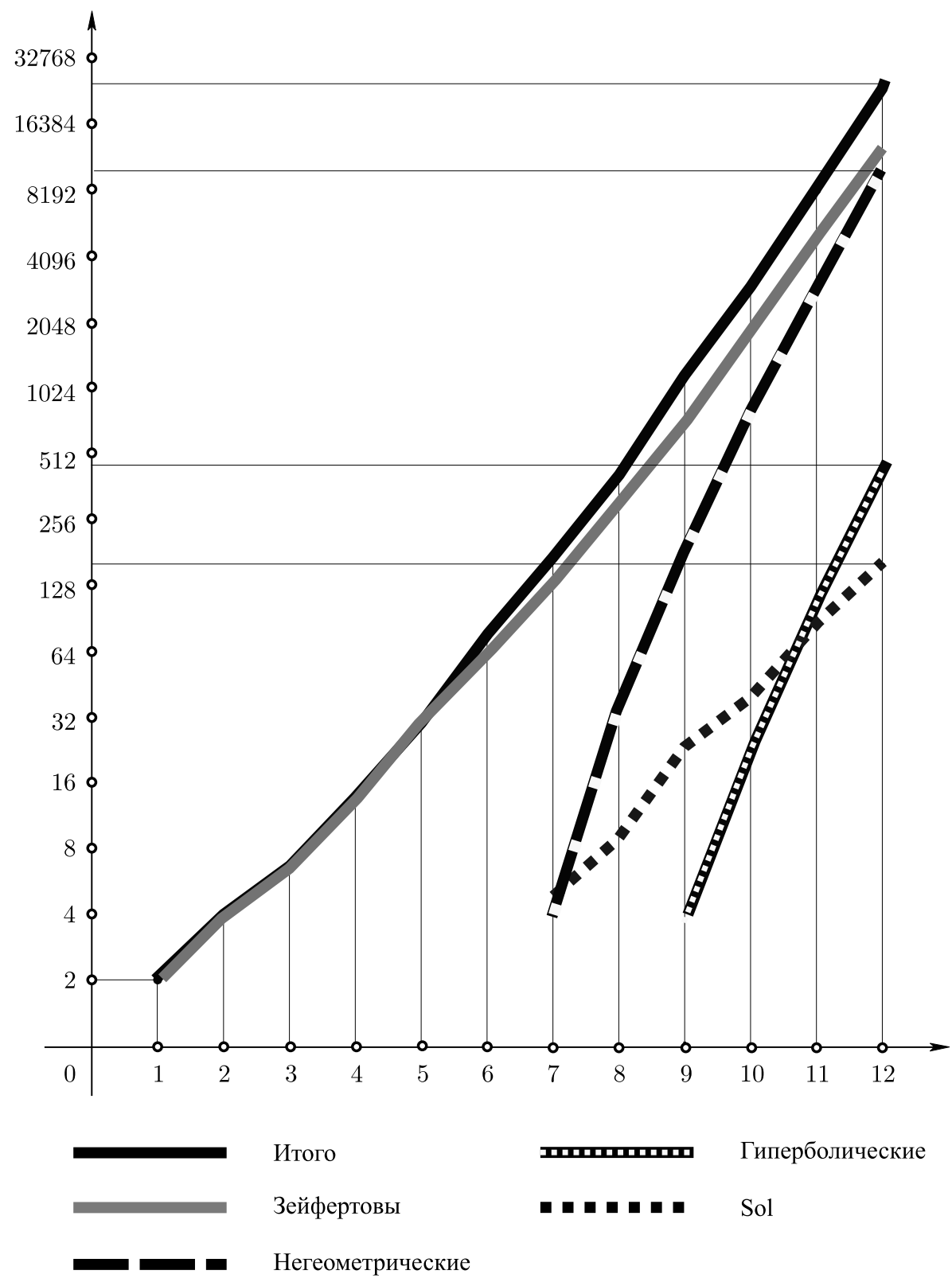

Рис. 7. Рост числа многообразий разных типов в зависимости от роста их сложности

Все многообразия до сложности 5 включительно являются эллиптическими и поэтому имеют конечные фундаментальные групш. В таблице представлены все пять известных серий конечных груп, которые могут свободно действовать на трехмерной сфере. Одно из самых интересных эллиптических многообразий, так назьваемое пространство додекаэдра (гомологическая сфера Пуанкаре) имеет сложность 5.

Все шесть плоских (т.е. обладаюших геометрией $E^{3}$ ) ориентируемых трехмерных 
многообразий расположены на уровне сложности 6. Там же расположены первые 7 Nil-многообразий. На уровне сложности 7 появляются 39 многообразий типа $\widetilde{S L_{2} \mathbb{R}}$, пять "оperatorname Sol и четыре составных многообразия. Каждое из этих составных многообразий разбивается несжимаемым тором на две части: утолщенную бутылку Клейна $K^{2} \tilde{\times} I=M\left(S^{2} ;(2,1),(2,-1)\right)$ и многообразие $M\left(S^{2} ;(2,1),(3,1)\right)$ (многообразия типа $K^{2} \tilde{\times} I \cup K^{2} \tilde{\times} I$ не являются составными, поскольку они допускают либо расслоение Зейферта, либо геометрическую структуру по образцу "оperatorname Sol).

Первые два многообразия $M\left(\mathbb{R} P^{2},(3,1),(3,-1)\right)$ и $M\left(S^{2},(2,1),(2,1),(3,1),(3,-4)\right)$ с геометрией $H^{2} \times \mathbb{R}$ расположены на уровне сложности 8 . Здесь уместно напомнить, что все замкнутые трехмерные многообразия сложности $\leqslant 8$ являются граф-многообразиями (этот факт доказан теоретически [14]). Структура составных (т.е. не являюшихся многообразиями Зейферта) графф-многообразий сложности $\leqslant 11$ очень проста. Есть всего три типа таких многообразий.

I. Два склеенных между собой многообразия Зейферта с базой диск и двумя или тремя особыми слоями.

II. Многообразие Зейферта $M\left(S^{1} \times I ;(\alpha, \beta)\right)$ с базой кольцо и одним особьм слоем, краевые торы которого заклеены многообразиями Зейферта с базой диск и двумя особыми слоями.

III. Многообразие Зейферта $M\left(S^{1} \times I ;(\alpha, \beta)\right)$, краевые торы которого склеены между собой.

Таким образом, графическая структура граф̆-многообразий до сложности 11 проста. Отвечаюшие им приведенные молекулы таковы: изолированная вершина, окружность с одной вершиной, отрезок и двухзвенная ломаная. На уровне сложности 12 появляются еше 5 типов молекул. Разумеется, 4 типа, встретившиеся на сложности $\leqslant 11$, сохраняются и на сложности 12 , см. рис. 8 .

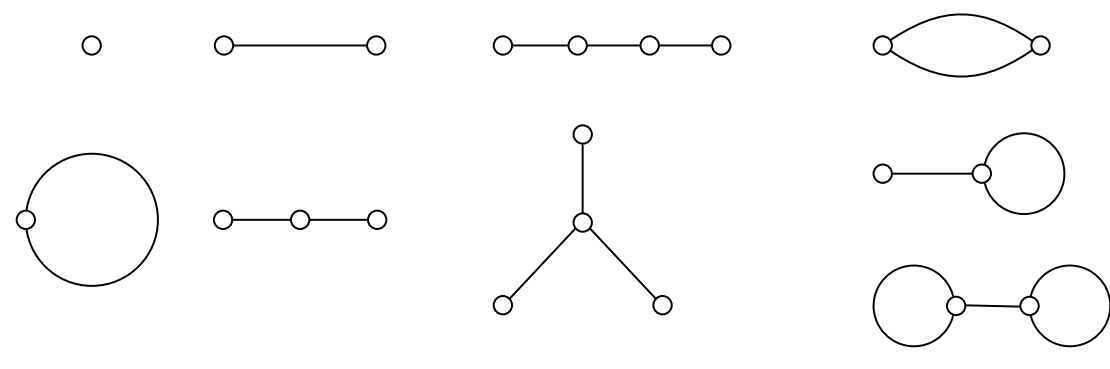

Рис. 8. Графическая структура многообразий сложности $\leqslant 12$

Первые многообразия, которые обладают гиперболической структурой и поэтому не являются граф-многообразиями, появляются на уровне сложности 9. О гиперболических многообразиях мы подробно расскажем в следующем пункте, а сейчас ограничимся упоминанием, что все негиперболические многообразия до сложности 11 , в JSJ-разбиении которых есть гиперболическая комната, устроены одинаково: они имеют вид $M^{2} \tilde{\times} S^{1} \cup Q_{1}$.

7.3. Гиперболические многообразия до сложности 11. Полная таблица замкнутых гиперболических многообразий до сложности 11 содержит 149 многообразий. Мы ограничимся ее небольшой частью, многообразиями сложности 9 и 10 , 
упорядочив их по объемам. В таблице 2 объемы приведены с тремя знаками после запятой.

ТЕОРема 5. Все замкнутье ориентируемые гиперболические трехмернье многообразия сложсности $\leqslant 10$ приведены в таблице 2.

ТАБлицА 2. Замкнутые гиперболические многообразия сложности $\leqslant 10$

\begin{tabular}{|c|c|c|}
\hline № & $H_{1}$ & объем \\
\hline 1 & $\mathbb{Z}_{5} \oplus \mathbb{Z}_{5}$ & 0.942 \\
\hline 2 & $\mathbb{Z}_{5}$ & 0.981 \\
\hline 3 & $\mathbb{Z}_{3} \oplus \mathbb{Z}_{6}$ & 1.014 \\
\hline 4 & $\mathbb{Z}_{5} \oplus \mathbb{Z}_{5}$ & 1.263 \\
\hline 5 & $\mathbb{Z}_{6}$ & 1.284 \\
\hline 6 & 0 & 1.398 \\
\hline 7 & $\mathbb{Z}_{6}$ & 1.414 \\
\hline 8 & $\mathbb{Z}_{10}$ & 1.414 \\
\hline 9 & $\mathbb{Z}_{35}$ & 1.423 \\
\hline 10 & $\mathbb{Z}_{3}$ & 1.440 \\
\hline 11 & $\mathbb{Z}_{5}$ & 1.529 \\
\hline 12 & $\mathbb{Z}_{21}$ & 1.543 \\
\hline 13 & $\mathbb{Z}_{35}$ & 1.543 \\
\hline $\mathbf{1 4}$ & $\mathbb{Z}_{40}$ & 1.583 \\
\hline 15 & $\mathbb{Z}_{21}$ & 1.583 \\
\hline
\end{tabular}

\begin{tabular}{|c|c|c|}
\hline № & $H_{1}$ & объем \\
\hline 16 & $\mathbb{Z}_{3} \oplus \mathbb{Z}_{9}$ & 1.583 \\
\hline 17 & $\mathbb{Z}_{30}$ & 1.588 \\
\hline 18 & $\mathbb{Z}_{30}$ & 1.588 \\
\hline 19 & $\mathbb{Z}_{5}$ & 1.610 \\
\hline 20 & $\mathbb{Z}_{7}$ & 1.649 \\
\hline 21 & $\mathbb{Z}_{15}$ & 1.649 \\
\hline 22 & $\mathbb{Z}_{7}$ & 1.757 \\
\hline 23 & $\mathbb{Z}_{3} \oplus \mathbb{Z}_{3}$ & 1.824 \\
\hline 24 & $\mathbb{Z}_{2} \oplus \mathbb{Z}_{12}$ & 1.831 \\
\hline 25 & $\mathbb{Z}_{7} \oplus \mathbb{Z}_{7}$ & 1.885 \\
\hline 26 & $\mathbb{Z}_{39}$ & 1.885 \\
\hline 27 & $\mathbb{Z}_{40}$ & 1.885 \\
\hline 28 & $\mathbb{Z}_{30}$ & 1.910 \\
\hline 29 & $\mathbb{Z}_{35}$ & 1.953 \\
\hline
\end{tabular}

Прокомментируем несколько интересных моментов, которые можно получить с помощью анализа как этой, так и полной электронной таблицы гиперболических многообразий.

1. Оказалось, что любое замкнутое гиперболическое многообразие до сложности 11 можно представить в виде $\left(Q_{i}\right)_{p, q}$, где $Q_{i}$ - гиперболические многообразия конечного объема с одним каспом, имеюшие сложность от 2 до 5. Все они содержатся в таблице многообразий с одним каспом, входящей в пакет программы SnapPea Дж. Уикса [29], см. также [18]. При этом большинство этих многообразий имеют по нескольку таких представлений. Например, многообразие Торстона $\left(Q_{2}\right)_{5,1}$ гомеоморфно многообразиям $\left(Q_{1}\right)_{1,2},\left(Q_{7}\right)_{1,1}$ и $\left(Q_{11}\right)_{2,-1}$. За счет таких гомеоморфизмов число необходимых гиперболических "кирпичей " $Q_{i}$ можно уменьшить. При анализе результатов компьютерного эксперимента выяснилось, что для представлений четырех замкнутых гиперболических многообразий сложности 9 достаточно двух кирпичей $Q_{1}$ и $Q_{2}$. Для сложности 10 нужно еще 9 кирпичей, а для сложности 11 - еше 12. 
2. Три табличных многообразия (их номера $14,19,20)$ пропущены в таблице замкнутых гиперболических многообразий Дж. Уикса, содержащейся в сопровождающем пакете программы "SnapPea". Более того, объем 1.610.. многообразия номер 19 также отсутствует в приведенном там монотонном списке объемов. Всего из 149 замкнутых гиперболических многообразий сложности $\leqslant 11$ потеряно 3 многообразия сложности 10 и 52 многообразия сложности 11. Скорее всего, Дж. Уикс вовсе и не ставил себе задачи составить список замкнутых гиперболических многообразий и объемов без пропусков, а просто упорядочил более или менее случайньй запас объемов, вычисленных к тому времени. Поэтому не удивительно, что систематический перебор в порядке возрастания сложности привел к новьм многообразиям и новым объемам.

3. Объем многообразия $\left(Q_{2}\right)_{7,-9}$ сложности 10 равен $1.649 \ldots$, тогда как многообразие $\left(Q_{2}\right)_{7,-8}$ сложности 11 имеет меньший объем $1.463 \ldots$ Это опровергает гипотезу о корелляии между сложностью трехмерного многообразия и его гиперболическим объемом (в рамках одной серии многообразий вида $Q_{p q}$ ), см. [18].

7.4. Проверка с помощью инвариантов. В процессе создания таблиц многообразий неоднократно появлялись (и публиковались) неточные результаты: в таблицах присутствовали дубликаты. Поэтому нами была произведена независимая проверка таблицы многообразий до сложности 11 на наличие дубликатов с помощью сравнения их первых групп гомологий и значений инвариантов Тураева-Виро [25] порядков $\leqslant 14$.

Инварианты вычислялись по программе, разработанной Е. Л. Первовой. При этом линзовые пространства были оставлены в стороне, поскольку вопрос о том, когда они имеют одинаковые инварианты Тураева-Виро, полностью решен [22]. Для того, чтобы обойти проблему приближенности компютерных вычислений, использовались не численные значения инвариантов, а их представления значениями полиномов с целыми коэффициентами в соответствуюших корнях из единищы. Такое совместное использование гомологий и инвариантов Тураева-Виро оказалось чрезвычайно эффективным: удалось различить все многообразия, за исключением 34 пар. Первая пара получается из многообразия Зейферта с базой кольцо и особым слоем типа $(2,1)$ склеиванием краев по гомеоморфизмам, задаваемым матрицами $\left(\begin{array}{cc}1 & 4 \\ 0 & -1\end{array}\right)$ и $\left(\begin{array}{cc}-1 & -4 \\ 0 & 1\end{array}\right)$. Вторая пара отличается только типом особого слоя, которьй задается параметрами $(3,2)$, и матрицами, которые записьваются так: $\left(\begin{array}{cc}1 & 3 \\ 0 & -1\end{array}\right)$ и $\left(\begin{array}{cc}-1 & -3 \\ 0 & 1\end{array}\right)$.

Совпадение всех инвариантов Тураева-Виро таких многообразий объясняется тем, что в обоих случаях одна матрица отличается от другой сменой знаков всех элементов. Этого достаточно для совпадения инвариантов всех (а не только вычисленных) порядков.

Многообразия всех остальных 32 пар являются многообразиями Зейферта, расслоенными над сферой с тремя особыми слоями. Вот пример такой пары: многообразия $M\left(S^{2},(2,1),(3,1),(6,1)\right)$ и $M\left(S^{2},(2,1),(3,1),(6,-11)\right)$ имеют одинаковые гомологии и одинаковые инварианты Тураева-Виро до порядка 14 (включая их гомологически тривиальные слагаемые, отвечаюшие целочисленньм раскраскам). Заметим, что первые параметры особых слоев этих многообразий одинаковы. Это наблюдение выполняется и для всех остальных пар: если инварианты Тураева-Виро не различают многообразия Зейферта указанного типа, то гомологии и первые инварианты особых 
слоев этих многообразий совпадают.

7.5. Классификация неориентируемых многообразий малой сложности. Первыми к табулированию замкнутых неориентируемых трехмерных многообразий обратились Г. Амендола и Б. Мартелли [1]. В отличие от ориентируемого случая, замкнутых неориентируемых $P^{2}$-неприводимых трехмерных многообразий сложности $\leqslant 5$ нет: неориентируемость заставляет многообразие быть сложнее. На сложности 6 есть ровно пять многообразий. Четыре из них обладают геометрией $E^{3}$, а пятое - геометрией Sol. Оно является многообразием Столлингса со слоем тор и матрицей монодромии $\left(\begin{array}{ll}1 & 1 \\ 1 & 0\end{array}\right)$. На уровне сложности 7 есть всего три замкнутых неориентируемых многообразия. Одно из них обладает геометрией Sol и расслаивается на торы с монодромией $\left(\begin{array}{ll}2 & 1 \\ 1 & 0\end{array}\right)$, а два других являются многообразиями Зейферта с геометрией $H^{2} \times \mathbb{R}$.

\section{8. Кристаллизации и расширенные диаграммы Хегора}

8.1. Геммы. Пусть $Г$ - регулярньй граф степени 4, ребра которого раскрашены четырьмя цветами так, что в каждой вершине сходятся ребра всех четырех цветов. Тогда пара $(\Gamma, \gamma)$, где $\gamma$-раскраска, называется геммой (от слов "graph-encoded manifold"). Если к Г приклеить 2-клетки по всем двухцветньм циклам, то получится специальньй спайн $P(\Gamma, \gamma)$ некоторого трехмерного многообразия $M=M(\Gamma, \gamma)$. Край многообразия $M$ всегда состоит из четырех непустых поверхностей (может быть, несвязных). Если он состоит из четырех сфер, то пара $(\Gamma, \gamma)$ называется кристаллизацией замкнутого многообразия $\widetilde{M}=\widetilde{M}(\Gamma, \gamma)$, получаюшегося из $M$ заклеиванием этих сфер шарами. Известно, что любое замкнутое трехмерное многообразие имеет кристаллизацию, см. работу [5], которая представляет собой хороший обзор по теории кристаллизаций, в том числе, многомерных многообразий.

ОПРЕДЕЛЕНИЕ 7 . Будем говорить, что кристаллизация $(\Gamma, \gamma)$ замкнутого ориентируемого трехмерного многообразия $M$ имеет род 2 , если для каких-нибудь двух цветов $a, b$ число покрашенных ими двухцветных циклов равно трем.

Число циклов кристаллизации рода 2, покрашенных двумя оставшимися цветами $c, d$, тоже равно 3 , тогда как других двухцветных циклов может быть и больше, и меньше. Объяснение простое: если из спайна $P(\Gamma, \gamma)$ удалить шесть 2-клеток, приклеенных по $a b$ - и $c d$-циклам, то получится замкнутая ориентируемая поверхность рода 2 . Она разбивает $M$ на два полных кренделя рода 2 , причем $a b$ - и $c d$-клетки служат меридиональными дисками этих кренделей и разбивают каждый из них на два шара. Поэтому не удивительно, что кристаллизации рода 2 очень близки к диаграммам Хегора рода 2.

\section{2. Расширенные диаграммы Хегора.}

ОПРЕДЕЛЕНИЕ 8. Система $\mathscr{M}=\mu_{1} \cup \mu_{2} \cup \mu_{3}$ непересекающихся простых замкнутых кривых на замкнутой ориентируемой поверхности $F$ рода 2 назьвается допустимой, если эти кривые разбивают $F$ на две связные части так, что к каждой кривой прилегают обе части. 
Несложное вычисление эйлеровой характеристики показьвает, что каждая часть в этом определении является сферой с тремя дырками.

ОПРЕДЕЛЕНИЕ 9. Расширенной диаграммой Хегора рода 2 называется тройка $(F, \mathscr{M}, \mathscr{L})$, где $F$ - замкнутая ориентируемая поверхность рода 2 , а $\mathscr{M}=\mu_{1} \cup \mu_{2} \cup \mu_{3}$, $\mathscr{L}=\lambda_{1} \cup \lambda_{2} \cup \lambda_{3}-$ две находящиеся в общем положении допустимые системы простых замкнутых кривых на ней.

Отметим, что если из систем $\mathscr{M}$ и $\mathscr{L}$ удалить по одной кривой, то получится обычная диаграмма Хегора. Обратно, к каждой обьчной диаграмме Хегора можно добавить пару кривых так, чтобы получилась расширенная диаграмма.

Как и обычные диаграммы, каждая расширенная диаграмма однозначно определяет некоторое замкнутое ориентируемое трехмерное многообразие $M$, которое можно построить следуюшим образом. Приклеим к поверхности $F \times\{0\} \subset F \times[0,1]$ ручки индекса 2 вдоль кривых первого семейства. Затем такие же ручки приклеим к поверхности $F \times\{1\} \subset F \times[0,1]$ вдоль кривых второго семейства. Край объединения многообразия $F \times[0,1]$ со всеми шестью приклеенными ручками будет состоять из четырех сфер. Заклеив их шарами, мы получим $M$.

Как было показано в конце предыдушего пункта, каждая кристаллизация рода 2 определяет расширенную диаграмму Хегора рода 2. Верно и обратное. По данной расширенной диаграмме рода 2 некоторого многообразия $M$ его кристаллизация строится так. Сопоставим четырем шарам, на которые меридиональные диски (средние диски ручек индекса 2 ) разбивают крендели, четыре цвета $a, b, c, d$. Обозначим через Г объединение краев меридиональных дисков, т.е. объединение $\mathscr{M} \cup \mathscr{L}$ кривых диаграммы. Тогда к каждому ребру графа Г примыкают ровно три разноцветных шара, и мы красим это ребро четвертым цветом.

8.3. Многообразия $M\left(n_{1}, n_{2}, n_{3}, k_{1}, k_{2}, k_{3}\right)$. Замкнутые ориентируемые многообразия рода 2 задаются так называемыми симметричнымми кристаллизациями рода, которые кодируются шестерками целых чисел вида $\left(n_{1}, n_{2}, n_{3}, k_{1}, k_{2}, k_{3}\right)$, см. [4]. Мы предпочитаем описать их на языке расширенных диаграмм Хегора.

Пусть $B$ - трехмерньй шар. Под треугольньм узором на сфере $\partial B$ мы понимаем набор $D_{1}, D_{2}, D_{3}$ когерентно ориентированных непересекающихся дисков в $\partial B$ вместе с набором $L$ соединяюших их дуг. Дуги не должны пересекаться, причем концы каждой дуги должны лежать на краях различных дисков.

Обозначим через $n_{i}$ число дуг, соединяющих $\partial D_{j}$ с $\partial D_{k}$, где $(i, j, k)$ - циклическая перестановка чисел $(1,2,3)$. Тогда общее число дуг в $L$ равно $n_{1}+n_{2}+n_{3}$. Мы будем называть объединение дисков $D_{i}$ с этими дугами треугольньм $\left(n_{1}, n_{2}, n_{3}\right)$-узором. Под примитивным топологическим поворотом диска $D_{i}$ понимается сохраняюший ориентацию гомеоморфизм $r_{i}: D_{i} \rightarrow D_{i}$, которьй переводит каждый конец дуги из $L \cap \partial D_{i}$ в конец следуюшей дуги (по отношению к положительному обходу окружности $\left.\partial D_{i}\right)$.

Мы построим зависяшее от шести параметров семейство расширенных диаграмм рода 2 следуюшим образом. Пусть $\left(n_{1}, n_{2}, n_{3}, k_{1}, k_{2}, k_{3}\right)$ - произвольная шестерка целых неотрицательных чисел. Возьмем два шара $B, B^{\prime}$ с одинаковыми треугольными $\left(n_{1}, n_{2}, n_{3}\right)$-узорами, расположенными симметрично относительно некоторой плоскости $\alpha$, см. рис. 9. 

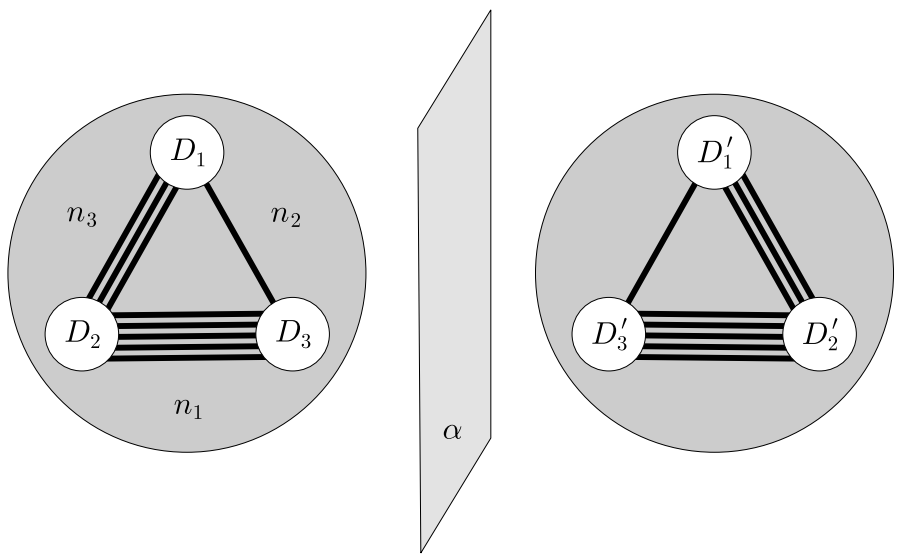

Рис. 9. Симметричные треугольные $\left(n_{1}, n_{2}, n_{3}\right)$-узоры

Обозначим через $s$ симметрию относительно этой плоскости. Мы склеиваем шары $B$ и $B^{\prime}$ путем отождествления каждого диска $D_{i}$ с симметричным ему диском $D_{i}^{\prime}$, $1 \leqslant i \leqslant 3$. Гомеоморфизм склеивания $h_{i}: D_{i} \rightarrow D_{i}^{\prime}$ задается правилом $h_{i}=s r_{i}^{k_{i}}$, где $s$ - симметрия и $r_{i}^{k_{i}}-k_{i}$-я степень примитивного топологического поворота. В peзультате такого склеивания получится полньй крендель $H$ рода 2 вместе с системой $\mathscr{L}=\lambda_{1} \cup \lambda_{2} \cup \cdots \cup \lambda_{m}$ нескольких простых замкнутых кривых на $\partial H$, склеенных из дуг $L$ и $L^{\prime}$. Обозначим через $\mathscr{M}=\mu_{1} \cup \mu_{2} \cup \mu_{3}$ набор окружностей в $\partial H$, полученных отождествлением краев дисков $D_{i}$ и $D_{i}^{\prime}$.

ОПРЕДЕЛЕНИЕ 10 . Шестерка чисел $\left(n_{1}, n_{2}, n_{3}, k_{1}, k_{2}, k_{3}\right)$ назьвается правильной, если

1) система $\mathscr{L}$ состоит из трех кривых и допустима;

2) после разрезания поверхности $F=\partial H$ по окружностям $\mathscr{L}=\lambda_{1} \cup \lambda_{2} \cup \lambda_{3}$ дуги, на которые при этом разрезаются меридианы $\mu_{1} \cup \mu_{2} \cup \mu_{3}$, образуют треугольный узор.

Из этого определения сразу следует, что если шестерка $\left(n_{1}, n_{2}, n_{3}, k_{1}, k_{2}, k_{3}\right)$ правильна, то тройка $(F, \mathscr{M}, \mathscr{L})$ является расширенной диаграммой Хегора рода 2 . Мы будем обозначать эту диаграмму через $D\left(n_{1}, n_{2}, n_{3}, k_{1}, k_{2}, k_{3}\right)$, а определяемое ею замкнутое ориентируемое трехмерное многообразие - через $M\left(n_{1}, n_{2}, n_{3}, k_{1}, k_{2}, k_{3}\right)$.

Теорема 6. Любая расширенная диаграмма Хегора $(F, \mathscr{M}, \mathscr{L})$ рода 2 әквивалентна диаграмме вида $D\left(n_{1}, n_{2}, n_{3}, k_{1}, k_{2}, k_{3}\right)$.

ДоКАЗАТЕЛЬСТВО. С помошью изотопии легко добиться, чтобы среди областей, на которые кривые $\mathscr{M}, \mathscr{L}$ разбивают поверхность $F$, не было двуугольников. Разрежем $F$ по кривьм $\mathscr{M}$. Если узор, составленньй из получившихся при этом дуг кривых $\mathscr{L}$ не является треугольным, то диаграмма упрощается с помощью волновых преобразований, описанных в [26], [19]. Аналогичные волновые преобразования упрошают диаграмму и в двойственном случае, когда кривые $\mathscr{M}$ и $\mathscr{L}$ меняются ролями. Выполняя такие упрошения до тех пор, пока это возможно, мы добьемся, чтобы оба узора были треугольными. 
Приведем итог вычислительного эксперимента по отбору правильных шестерок и распознаванию отвечающих им многообразий.

ТеОрема 7. Существует ровно 176 замкнутых ориентируемых неприводимых трехмерных многообразий рода 2, допускающих кристаллизации с не более чем 48 вершинами. Среди них есть 7 многообразий, обладающих геометрией Sol, 5 гиперболических многообразий и 6 многообразий, не допускающих однородной геометрии. Все остальные многообразия являются многообразиями Зейферта.

Этот результат сильнее теоремы П. Бандиери, К. Гальярди, Л. Риччи [2] о существовании ровно 26 замкнутых неприводимых многообразий рода 2 , имеющих кристаллизации с не более чем 34 вершинами.

\section{СПИСОК ЛИТЕРАТУРЫ}

[1] G. Amendola, B. Martelli. Non-orientable 3-manifolds of complexity up to 7 // Topology Appl. 2003. V. 133. № 2. P. 157-178.

[2] P. Bandieri, C. Gagliardi, L. Ricci. Classifying genus two 3-manifolds up to 34 tetrahedra // Acta Appl. Math. 2005. V. 86. № 3. P. 267-283.

[3] P. J. Callahan, M.V. Hildebrand, J.R. Weeks. A census of cusped hyperbolic 3-manifolds // Math. Comp. 1999. V. 68. № 225. P. 321-332.

[4] M. R. Casali, L. Grasselli. 2-symmetric crystallizations and 2-fold branched coverings of $S^{3}$ // Discrete Math. 1991. V. 87. №1. P. 9-22.

[5] M. Ferri, C. Gagliardi, L. Grasselli. A graph-theoretical representation of PL-manifolds - a survey on crystallizations // Aequationes Math. 1986. V. 31. № 2-3. P. 121-141.

[6] W. Jaco, P. B. Shalen. A new decomposition theorem for irreducible sufficiently large 3-manifolds // Algebraic and Geometric Topology (Stanford, 1976). Part 2. Providence, RI: Amer. Math. Soc., 1978. P. 71-84. (Proc. Sympos. Pure Math. V. 32.)

[7] W. H. Jaco, P. B. Shalen. Seifert fibered spaces in 3-manifolds // Mem. Amer. Math. Soc. 1979. V. 21. № 220.

[8] K. Johannson. Homotopy Equivalences of 3-manifolds with Boundaries. Berlin: SpringerVerlag 1979. (Lecture Notes in Math. V. 761.)

[9] S. Lins. Gems, Computers and Attractors for 3-manifolds. River Edge: World Scientific, 1995. (Knots Everything. V. 5.)

[10] B. Martelli. Complexity of 3-manifolds // ArXiv: math.GT/0405250.

[11] B. Martelli, C. Petronio. Three-manifolds having complexity at moxt 9 // Experiment. Math. 2001. V. 10. № 2. P. 207-236.

[12] С. В. Матвеев, В.В. Савватеев. Трехмерные многообразия, имеющие простые специальные спайны // Colloq. Math. 1974. V. 32. P. 83-97.

[13] С. В. Матвеев. Сложность трехмерных многообразий и их перечисление в порядке возрастания сложности // Докл. АН СССР. 1988.. Т. 301. № 2. С. 280-284.

[14] S. Matveev. Complexity theory of three-dimensional manifolds // Acta Appl. Math. 1990. V. 19. № 2. P. 101-130.

[15] S. V. Matveev. Large tables of 3-manifolds up to complexity 6 // Preprint MPI 1998-67. Bonn: Max Planck Institute for Mathematics, 1998.

[16] S. Matveev. Algoritghmic Topology and Classification of 3-manifolds. Berlin: Springer-Verlag, 2003. (Algorithms Comput. Math. V. 9.)

[17] С. В. Матвеев. Распознавание и табулирование трехмерных многообразий // Докл. PAH. 2005. T. 400. № 1. C. 26-28.

[18] С. В. Матвеев, А. Т. Фоменко. Изоэнергетические поверхности интегрируемых гамилтоновых систем, перечисление трехмерных мноообразий в порядке возрастания их сложности и вычисление объемов замкнутых гиперболических многообразий // УМН. 1988. T. 43. № 1. C. 5-22. 
[19] С. В. Матвеев, А. Т. Фоменко. Алгоритмические и компьютерные методы в трехмерной топологии. М.: Наука, 1998.

[20] А. М. Овчинников. Построение специальных сплайнов трехмерных многообразий Вальдхаузена // Дисс. ... канд. физ.-матем. наук. Челябинск: ЧелГУ, 2000.

[21] П. Скотт. Геометрии на трехмерных многообразиях. М.: Мир, 1986.

[22] М.В. Соколов. Какие линзовые пространства различаются инвариантами Тураева-Виро // Матем. заметки. 1997. Т. 61. № 3. С. 468-470.

[23] W. Thurston. Three-dimensional manifolds, Kleinian groups and hyperbolic geometry // Bull. Amer. Math. Soc. 1982. V. 6. № 3. P. 357-381.

[24] У. Тёрстон [Торстон]. Трехмерная геометрия и топология на трехмерных многообразиях. М.: МЦНМО, 2001.

[25] V. G. Turaev, O. Y. Viro. State sum invariants of 3-manifolds and quantum $6 j$-symbol // Topology. 1992. V. 31. №4. P. 865-902.

[26] И. А. Володин, В. Е. Кузнецов, А. Т. Фоменко. О проблеме алгоритмического распознавания стандартной трехмерной сферы // УМН. 1974. Т. 29. № 5. С. 71-168.

[27] F. Waldhausen. Eine Klasse von 3-dimensionalen Mannigfaltigkeiten. I, II // Invent. Math. 1967. V. 3. P. 308-333; V. 4. P. 87-117.

[28] J. Weeks. Hyperbolic Structures on 3-manifolds // Ph.D. Thesis. Princeton: Princeton University, 1985.

[29] J. Weeks. SnapPea, A computer program for creating and studying hyperbolic 3-manifolds // available for anonymous ftp from geom.umn.edu/pub/sofware/snappea.

Челябинский государственньй университет

Поступила в редакцию

E-mail: matveev@csu.ru

11.05 .2005 